\title{
Collective Lamb Shift of a Nanoscale Atomic Vapor Layer within a Sapphire Cavity
}

\author{
T. Peyrot, Y. R. P. Sortais, and A. Browaeys \\ Laboratoire Charles Fabry, Institut d'Optique Graduate School, CNRS, Université Paris-Saclay, \\ F-91127 Palaiseau Cedex, France \\ A. Sargsyan and D. Sarkisyan \\ Institute for Physical Research, National Academy of Sciences, Ashtarak 2, 0203, Armenia \\ J. Keaveney, I. G. Hughes, and C. S. Adams \\ Department of Physics, Rochester Building, Durham University, South Road, Durham DH1 3LE, United Kingdom
}

(Received 22 December 2017; revised manuscript received 17 April 2018; published 12 June 2018)

\begin{abstract}
We measure the near-resonant transmission of light through a dense medium of potassium vapor confined in a cell with nanometer thickness in order to investigate the origin and validity of the collective Lamb shift. A complete model including the multiple reflections in the nanocell reproduces accurately the observed line shape. It allows the extraction of a density-dependent shift and width of the bulk atomic medium resonance, deconvolved from the cavity effect. We observe an additional, unexpected dependence of the shift with the thickness of the medium. This extra dependence demands further experimental and theoretical investigations.
\end{abstract}

DOI: 10.1103/PhysRevLett.120.243401

When many light emitters are subjected to an electromagnetic field with a wavelength $\lambda$, they may react collectively to the field $[1,2]$. A well-known example of collective response is the enhancement of the decay rate of an atomic ensemble with respect to the individual atom case. Owing to the coupling of atoms via resonant dipole-dipole interactions, it becomes important when the volume of interaction is smaller than $(\lambda / 2 \pi)^{3}$. Collective effects in light scattering have gained a renewed interest recently with the recognition that they can bias the accuracy of atom-based sensors such as optical clocks by introducing unwanted energy level shifts [3-5]. Alternatively, the collective response can be an asset if properly handled, and several recent works suggest how it can be used to enhance light-matter interfaces [6-9].

The resonant dipole-dipole interactions between atoms should lead to a collective frequency shift of the atomic lines [10]. This shift, unfortunately named the cooperative or collective Lamb shift (CLS) despite its classical nature, depends on the shape of the sample. In the case of an atomic slab of thickness $L$ and density $N$, it was predicted to be [10]

$$
\Delta_{\mathrm{CLS}}=\Delta_{\mathrm{LL}}-\frac{3}{4} \Delta_{\mathrm{LL}}\left(1-\frac{\sin 2 k L}{2 k L}\right)
$$

Published by the American Physical Society under the terms of the Creative Commons Attribution 4.0 International license. Further distribution of this work must maintain attribution to the author(s) and the published article's title, journal citation, and DOI. where $\Delta_{\mathrm{LL}}=-\pi\left(N / k^{3}\right) \Gamma$ is the Lorentz-Lorenz shift, $k=2 \pi / \lambda$ is the wave vector, and $\Gamma$ is the natural linewidth of the relevant atomic transition. Four decades later, the first measurements of the CLS were reported using a layer of Fe atoms [11] and a slab of hot alkali vapor [12]. Following these experiments, it was pointed out that Eq. (1) is valid only in the low-density limit $\left(N / k^{3} \ll 1\right.$ with $N$ the density of the vapor $[13,14])$, a condition not met by the experiment of Ref. [12] for which $N / k^{3} \sim 100$. Reference [15] suggested that this CLS should be present only when large inhomogeneous broadening is present, such as in hot vapor. However, subsequent experiments on ultracold atoms (insignificant inhomogeneous broadening) reported either a shift consistent with the CLS prediction [16] or a negligible shift [17]. Recently, theoretical work highlighted that the CLS in a slab geometry [13] should merely arise from cavity interferences between the boundaries of the medium. In contrast to the original suggestion [10], in the cavity viewpoint, the CLS would not be related to the Lorentz local field. Clearly, the situation is confusing, and further work is needed to clarify it.

In this Letter, we present a new investigation of the origin and validity of the CLS. To do so, we measure the transmission resonance line shape of a dense hot vapor of potassium atoms confined in a slab with nanometer thickness. We develop a new model to interpret the data based on standard mean-field electromagnetism. It includes the multiple reflections due to the cavity formed by the two layers of sapphire enclosing the atomic vapor. We show, in particular, that Eq. (1) is valid only in the limit of a 
low-density atomic slab surrounded by a vacuum, neither conditions being fulfilled here. Furthermore, using the model, we deconvolve the cavity effect from the measured transmission and extract the shift of the atomic resonance line as a function of the density and thickness. We observe an unexpected oscillatory dependence of the shift with the slab thickness, which indicates that further refinement of the theory is needed in order to fully account for the optical properties of dense media.

We first give a simple derivation of the CLS [Eq. (1)] that highlights the roles of the boundaries and of the dipoledipole interactions between atoms, as well as its range of applicability. We consider an atomic slab (thickness $L$, susceptibility $\chi$, refractive index $n=\sqrt{1+\chi}$ ) placed in vacuum and illuminated by a plane wave $E_{0} \exp [i k z]$ with frequency $\omega=c k$. As the light propagates in the medium, the fields radiated by the induced dipoles interfere with the incident field and, in turn, excite new atoms: The dipoledipole interaction, which is the interaction of the field radiated by an atomic dipole with another dipole [18], is thus included in the description of the propagation. The field scattered at position $z$ by a slice of thickness $d z^{\prime} \ll \lambda$ located at position $z^{\prime}$ is $d E_{\mathrm{sc}}(z)=i k P\left(z^{\prime}\right) /\left(2 \epsilon_{0}\right) \exp [i k \mid z-$ $\left.z^{\prime} \mid\right] d z^{\prime}[18,19]$. Here $P\left(z^{\prime}\right)$ is the polarization vector related to the total field $E\left(z^{\prime}\right)$ inside the medium by $P\left(z^{\prime}\right)=$ $\epsilon_{0} \chi E\left(z^{\prime}\right)$. Consequently, the superposition principle yields the field transmitted by the slab:

$$
E_{t}(z>L)=E_{0} e^{i k z}+\frac{i k \chi}{2} \int_{0}^{L} E\left(z^{\prime}\right) e^{i k\left(z-z^{\prime}\right)} d z^{\prime} .
$$

To calculate the total field inside the slab, we neglect the multiple reflections at the boundaries between the medium and vacuum. Therefore, $E\left(z^{\prime}\right) \approx t E_{0} e^{i n k z^{\prime}}+r t E_{0} e^{i n k\left(2 L-z^{\prime}\right)}$, with $n \approx 1+\chi / 2, t=2 /(n+1) \approx 1-\chi / 4$, and $r=(n-1) /$ $(n+1) \approx \chi / 4$, for $\chi \ll 1$. Using these expressions in Eq. (2), we get, up to second order in $\chi$,

$E_{t} \approx E_{0} e^{i k z}\left[1+i \frac{\chi k L}{2}\left(1+i \frac{\chi k L}{4}-\frac{\chi}{4}+\frac{\chi}{4} \frac{e^{2 i k L}-1}{2 i k L}\right)\right]$.

The susceptibility of the dilute slab consisting of atoms with polarizability $\alpha=i\left(6 \pi \Gamma / k^{3}\right) /\left(\Gamma_{t}-2 i \Delta\right)\left(\Delta=\omega-\omega_{0}\right.$ with $\omega_{0}$ the resonant frequency, $\Gamma$ the radiative linewidth, and $\Gamma_{t}$ the total homogeneous linewidth) is $\chi=N \alpha$. Using $1+x \approx 1 /(1-x)$ for $|x| \ll 1$ in the parentheses in Eq. (3), we obtain the transmission coefficient:

$$
T(\Delta)=\left|\frac{E_{t}}{E_{0}}\right|^{2}=\left|1-\frac{3 \pi N L}{k^{2}} \frac{\Gamma}{\Gamma_{c}-2 i\left(\Delta-\Delta_{c}\right)}\right|^{2},
$$

with the thickness-dependent shift $\Delta_{c}=-\frac{3}{4} \Delta_{\mathrm{LL}}[1-$ $(\sin 2 k L / 2 k L)]$ and $\Gamma_{c}=\Gamma_{t}-\frac{3}{4}\left[k L+\left(\sin ^{2} k L / k L\right)\right] \Delta_{\mathrm{LL}}$. The offset $-\frac{3}{4} \Delta_{\mathrm{LL}}$ in the shift is traced back to the transmission through the first interface. To recover the extra offset $\Delta_{\mathrm{LL}}$ in Eq. (1), we must use the Lorentz-Lorenz formula [20] in Eq. (3): $\chi=N \alpha /(1-N \alpha / 3)$. This derivation therefore shows that (i) the CLS is a frequency shift of the position of the transmission minimum and not a shift of the resonance $\omega_{0}$ of the bulk medium characterized by $\chi$, (ii) it is a consequence of the reflection of the field at the boundaries of the slab, (iii) it includes the dipoledipole interactions in the propagation, and (iv) Eq. (1) is valid only in a medium for which $\chi \ll 1$ at resonance, i.e., $\left(N / k^{3}\right)\left(\Gamma / \Gamma_{t}\right) \ll 1$.

To extend the model beyond the dilute regime, we include the multiple reflections in the cavity produced by the interface between the atomic medium and its environment (index $n_{s}$ ). Using a textbook interference argument [13], we calculate the transmission coefficient of the field amplitude and get

$$
t(\Delta)=\frac{4 n_{s} n \exp \left[i\left(n-n_{s}\right) k L\right]}{\left(n_{s}+n\right)^{2}-\left(n_{s}-n\right)^{2} \exp [2 i n k L]} .
$$

For $n_{s}=1$, Eq. (5) predicts that the frequency of the minimum transmission $\Delta_{\min }$ does follow Eq. (1) but only when $\left(N / k^{3}\right)\left(\Gamma / \Gamma_{t}\right) \ll 1$ (see Fig. 4 in Ref. [14]). This is no longer true when the atomic medium is confined between two windows of index $n_{s} \neq 1$, as is the case for nanocells: There, $\Delta_{\min }$ never follows Eq. (1) even at a low density (see details in Ref. [21]).

We now describe our experimental investigation of the CLS using a nanocell [24]. The nanocell [Fig. 1(a)] consists of two 1-mm-thick sapphire wedge plates $\left(n_{s}=1.76\right)$ filled with a vapor of potassium [25]. The resulting atomic slab has a thickness $L$ varying between $50 \mathrm{~nm}$ and $1.5 \mu \mathrm{m}$ [26]. The atomic density is controlled by heating the cell from room temperature up to $380^{\circ} \mathrm{C}$, achieving similar densities as in Ref. [12]. Compared to the earlier measurements performed in rubidium [12], potassium has the advantage of a smaller hyperfine splitting in the ground state, which results into a single atomic line at lower densities. The optical setup is shown in Fig. 1(b). We measure the transmission of a laser beam nearly resonant with the $D 2$ transition of ${ }^{39} \mathrm{~K}(\lambda \approx 767 \mathrm{~nm}, \Gamma=2 \pi \times 6 \mathrm{MHz})$. The beam is produced by a commercial external cavity laser diode, focused on the cell sapphire windows with a waist $w \approx 40 \mu \mathrm{m} \gg L$. We use the interferometric techniques described in Ref. [27] to measure the local thickness. The laser is scanned across the resonance over a range of about $30 \mathrm{GHz}$. The intensity is stabilized using a PID-controlled acousto-optic modulator [28]. The frequency of the laser is calibrated by standard saturated absorption spectroscopy in a $7.5 \mathrm{~cm}$ potassium reference cell.

Figure 1(c) shows the measured optical density (OD), extracted from the transmission $T$ via $\mathrm{OD}=-\ln (T)$, as a function of the laser detuning $\Delta$ for three values of the atomic density $N$. We plot $\Delta_{\text {min }}$, defined as the detuning at which the OD is the largest, as a function of the density for 
(a)

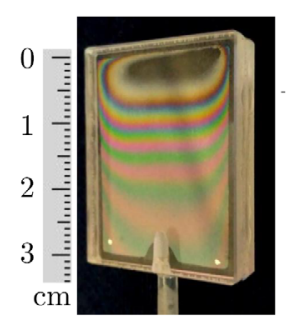

(c)

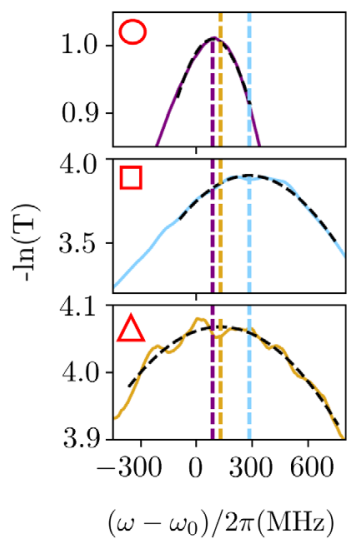

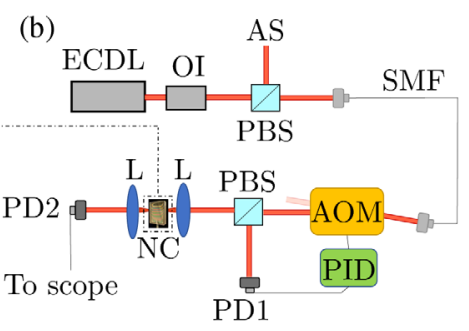

(d)

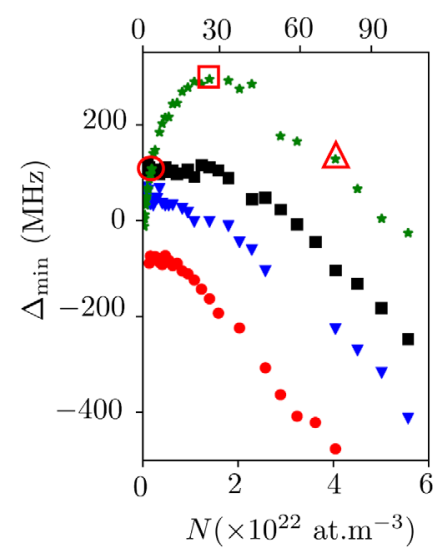

FIG. 1. (a) Nanocell used in the experiment. The interference fringes indicate that the thickness of the slab between the two sapphire windows varies from $50 \mathrm{~nm}$ to $1.5 \mu \mathrm{m}$ at the bottom. (b) Optical setup. ECDL, external cavity laser diode; OI, optical isolator; PBS, polarization beam splitter; SMF, single-mode fiber; NC, nanocell; PD, photodiode; AOM, acousto-optic modulator; $L$, lens; AS, absorption spectroscopy; PID, proportional-integralderivative controller. (c) Measured optical density for a slab of thickness $L=490 \mathrm{~nm}$ as a function of the detuning and for temperatures $\Theta=\left(260^{\circ} \mathrm{C}, 340{ }^{\circ} \mathrm{C}, 380^{\circ} \mathrm{C}\right.$ ) (top, middle, and bottom, respectively), corresponding to $N / k^{3}=(3,29,74)$. Vertical dotted lines: Frequency $\Delta_{\min }$ of the maximum of the OD. (d) $\Delta_{\text {min }}$ versus density $N$ for $L=90 \mathrm{~nm}$ (red dots), $L=$ $110 \mathrm{~nm}$ (blue triangles), $L=\lambda / 2=380 \mathrm{~nm}$ (black squares), and $L=490 \mathrm{~nm}$ (green stars). The empty square, triangle, and circle correspond to the curves in (c).

various thicknesses $L$ in Fig. 1(d). At a high density $\left(N / k^{3} \gtrsim 20\right)$, we observe a redshifted, linear variation of $\Delta_{\min }$ with $N$ for all $L$. At low $N$, for $L>\lambda / 2, \Delta_{\min }$ exhibits a pronounced blueshift and turns into a redshift at a higher density. For thicknesses $L \lesssim \lambda / 2, \Delta_{\min }$ features a plateau at low $N$, as also seen in Ref. [12]. Similar blueshifts of the minimal transmission were observed in a nanocell of cesium [29], although much smaller than here, and recently in a slab of ultracold rubidium atoms [17], where an evolution from the blue to the red side of the resonance was also measured.

To explain the data, we now develop a model that deconvolves the effect of the cavity produced by the interface between the sapphire windows and the atomic medium and the bulk properties of the atomic medium. This was also the approach used in Ref. [12]. However, the

model used there to extract the shift took only partially the cavity effect into account (see details in Ref. [21]). Furthermore, as explained above, Eq. (1) is irrelevant for the experimental situation of a nanocell: The atomic slab should be dilute and surrounded by a vacuum for the formula to hold. The agreement between the measured shift as a function of the cell thickness and Eq. (1) must therefore be considered as fortuitous.

Our new model incorporates the multiple reflections in the cavity by using Eq. (5). As for the atomic slab, it is described by a continuous resonant medium with a refractive index $n$. Ascribing a refractive index to a hot vapor confined in a nanocell is far from being obvious, as has been studied in great detail (e.g., Refs. [30-33]). First, the Doppler effect leads to a nonlocal refractive index, and, second, the small thickness of the cell results in a nonsteady-state response of most atoms but the ones flying parallel to the cell surface. However, when the density is as large as the ones used here, the collisional broadening of the line $\Gamma_{p}$ exceeds the Doppler width $\Delta \omega_{D}$ [see below and Fig. 2(d)]: The atomic dipoles reach their steady state over a distance $\sim \Delta \omega_{D} /\left(k \Gamma_{p}\right)$, much smaller than $L$ and $\lambda$. It then becomes possible to define a steady-state, local refractive index [31].

We relate the refractive index of the atomic slab to the electric susceptibility $\chi$ by $n(\Delta)=\sqrt{1+\chi(\Delta)}$. Here we take $\chi=N \alpha_{p}$ with $\alpha_{p}(\Delta, N)$ the polarizability of the atoms, including the influence of the density at the single atom level through a broadening and a shift. It is calculated by summing the contribution of all hyperfine transitions of the $D 2$ line with Lorentzian profiles, weighted by the corresponding Clebsch-Gordan coefficients [34] ( $\Gamma$ is the radiative decay rate of the strongest transition):

$$
\alpha_{p}(\Delta, N)=i \frac{6 \pi \Gamma}{k^{3}} \sum_{F, F^{\prime}} \frac{C_{F F^{\prime}}^{2}}{\Gamma_{t}-2 i \Delta_{t}} .
$$

Here, we do not integrate over the velocity distribution, as Doppler broadening is negligible with respect to the homogeneous broadening [35]. In Eq. (6), $\Gamma_{t}=\Gamma+\Gamma_{p}$ is the sum of the radiative linewidth $\Gamma$ and a width $\Gamma_{p}$ that accounts in a phenomenological way for any broadening mechanism inside the gas beyond the cavity-induced broadening. In the same way, the detuning $\Delta_{t}=$ $\Delta+\Delta_{F F^{\prime}}+\Delta_{p}$, with $\Delta_{F F^{\prime}}$ the hyperfine splitting and $\Delta_{p}$ a phenomenological shift inside the gas beyond the cavity-induced shift. The quantities $\Delta_{p}(N, L)$ and $\Gamma_{p}(N, L)$ therefore contain the physics not included in the model: (i) the interaction of the atoms with the cell walls (dependent on only the thickness $L$ ), (ii) the collisional dipole-dipole interactions between the light-induced dipoles (dependent on only the density $N$ ), and (iii) any extra effects that may depend on both $L$ and $N$. Finally, to compare our model to the data, we normalize the 

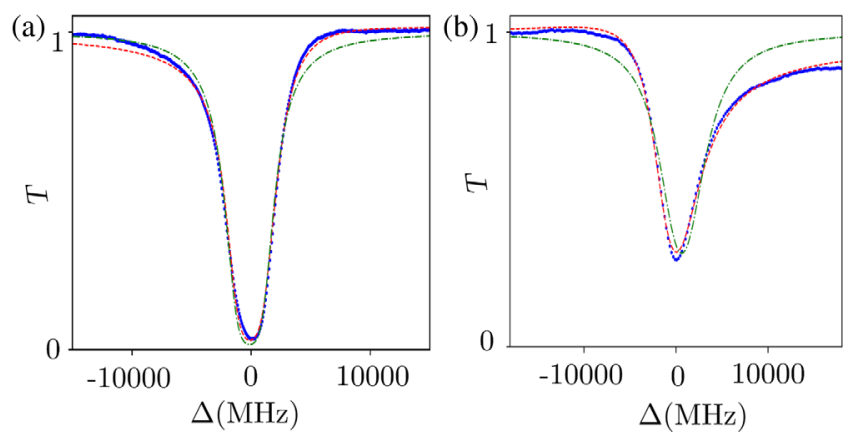

(c)

(d)
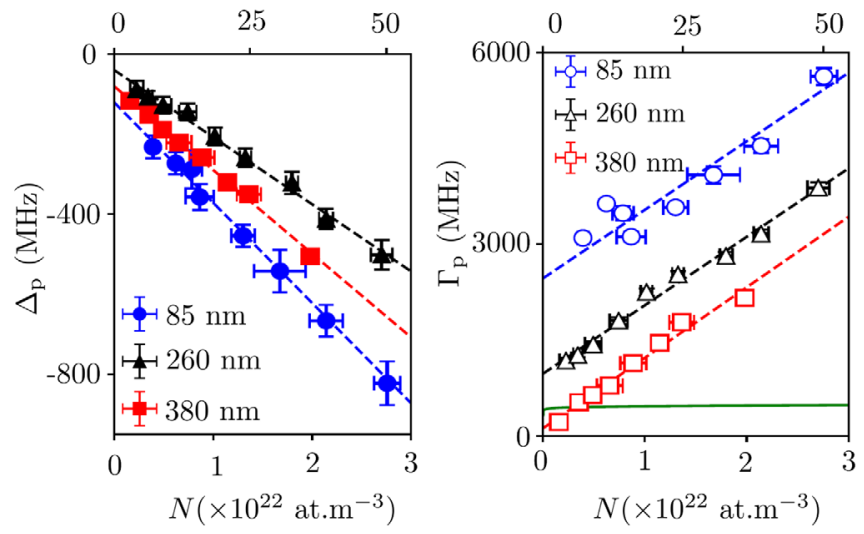

FIG. 2. Top panels represent transmission profiles for (a) $\Theta=$ $330^{\circ} \mathrm{C}$ and $L=440 \mathrm{~nm}$ and (b) $\Theta=365^{\circ} \mathrm{C}$ at $L=\lambda / 4$, where the asymmetry is most pronounced. Blue dots: Measured transmission. Green line: Transmission calculated with the model where $n_{s}=1$. Red dashed line: Transmission calculated by the cavity model where $n_{s}=1.76$. (c) Experimental bulk shift $\Delta_{p}$ and (d) broadening $\Gamma_{p}$ (deconvolved from the cavity) for various cell thicknesses $L$. Solid line: Doppler width. The dashed lines are linear fits to the data. The error bars on both axes are extracted from the fit.

transmission coefficient in intensity to the nonresonant case $(n=1): T=|t(\Delta) / t(\Delta \rightarrow \infty)|^{2}$.

Figures 2(a) and 2(b) show a comparison of the model's prediction and the measured line shape. The agreement is very good. In particular, the model reproduces the observed asymmetric line shape and the blueshift of the maximum optical depth observed in Fig. 1(d) (see more details in Ref. [21]). To demonstrate the importance of the sapphire layers in the optical response, we also plot in Fig. 2(a) the result of Eq. (5) for the case of an atomic layer immersed in a vacuum $\left(n_{s}=1\right)$ : There, the asymmetry is nearly absent.

To fit the data by the model and obtain the good agreement shown in Figs. 2(a) and 2(b), we let the density $N$ (or, equivalently, the temperature $\Theta$ [36]), the line shift $\Delta_{p}$, and the broadening $\Gamma_{p}$ be free parameters. In Figs. 2(c) and 2(d), we plot the fitted values of $\Delta_{p}$ and $\Gamma_{p}$ as a function of the fitted $N$, for various thicknesses. Both $\Delta_{p}$ and $\Gamma_{p}$ have an offset at an asymptotically low density that increases when the thickness of the cell decreases. Its origin lies in the interaction between the atoms and the walls of the nanocell, as was measured in Ref. [37]: When the thickness decreases, the fraction of atoms interacting significantly with the cell walls increases. Figure 2(d) indicates that $\Gamma_{p}$ is much larger than the Doppler width and the broadening is dominated by the density-dependent contribution coming from the collisional dipole-dipole interactions. For the range of densities explored here, the vapor is thus homogeneously broadened with $\left(N / k^{3}\right)\left(\Gamma / \Gamma_{t}\right) \lesssim 1$.

To remove the influence of the surface on the shift (an effect depending on $L$ only), we fit the data presented in Figs. 2(c) and 2(d) by a linear function and extract the slopes $\left(\partial \Delta_{p} / \partial N\right)(L)$ and $\left(\partial \Gamma_{p} / \partial N\right)(L)$. We plot in Figs. 3(a) and 3(b) these slopes as a function of the thickness $L$. Both quantities feature an offset that we attribute to collisional dipole-dipole interactions between atoms. For example, the offset on $\partial \Gamma_{p} / \partial N$ extracted from Fig. 2(d) is close to the calculated self-broadening coefficient resulting from the collisional dipole-dipole interactions $\beta=2 \pi \sqrt{2} \Gamma / k^{3}$ [38] [dotted line in Fig. 3(b)]. However, we also observe a residual oscillation of the shift slope with a period $(0.5 \pm 0.02) \lambda$ (the error bars are discussed in Ref. [21]). This oscillation is unexpected: All known dependences of $\Delta_{p}$ with the cell thickness are included and should result in a shift slope being a bulk property of the medium, i.e., independent of $L$.

We finally examine possible explanations for the residual shift shown in Fig. 3(a). A first possibility could

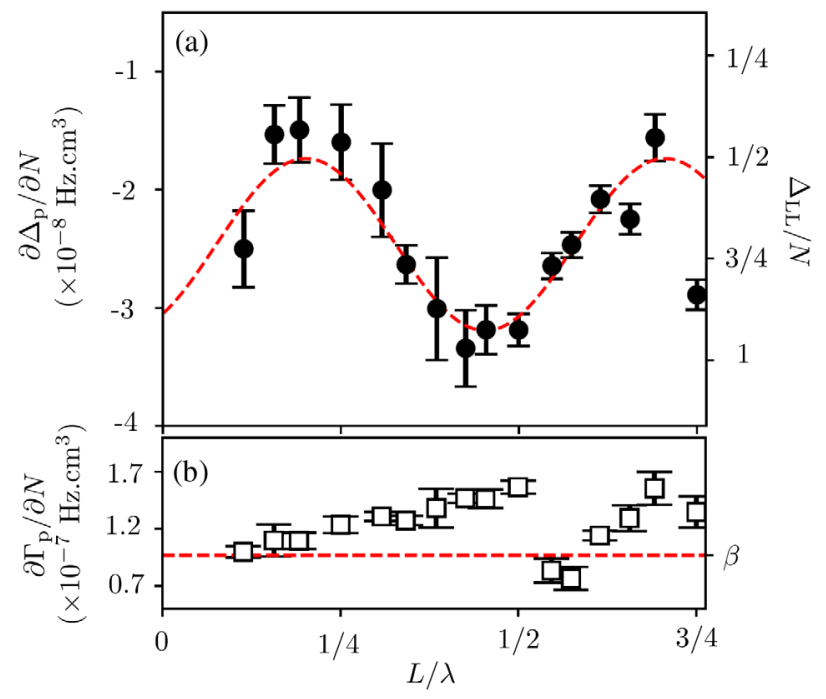

FIG. 3. (a) Black dots: Slope $\partial \Delta_{p} / \partial N$ of the shift extracted from the cavity model as a function of the cell thickness $L$, together with the fit by a sinusoidal function (dashed red line). (b) Black empty squares: Slope $\partial \Gamma_{p} / \partial N$ of the width extracted from the cavity model as a function of $L$. The red dashed line is the theoretical value of the self-broadening coefficient $\beta$ resulting from the collisional interactions between atoms (see the text). The errors bars are dominated by the systematic effects detailed in Ref. [21]. 
be a non-Maxwellian velocity distribution due to the cell surface [39]. Dicke coherent narrowing [40], which depends on the cell thickness, is also expected in nanocells. However, the measured linewidth $\Gamma_{p}$ is much larger than the expected Doppler width, and any modifications of the velocity distribution should have a negligible effect on the extracted value of $\Delta_{p}$. A second possibility could be the influence of the correlations among dipoles induced by the interactions. They are ignored in our treatment of the configuration-averaged field and in all the models developed so far [10,13-15,41,42]. This assumption is valid for dilute gases, but it could fail at higher densities such as the ones explored here. Going beyond a mean-field approach by including them could lead to a nonlocal response of the gas. The models presented here or in Refs. [10,13-15], which assume a local susceptibility, would then fail-and including the correlations would be a highly nontrivial undertaking.

In conclusion, we have performed a new series of measurements of the transmission of near-resonant light through an alkali vapor with nanometer scale thickness in order to investigate the origin and validity of the collective Lamb shift. A model, deconvolving the cavity effect from the atomic properties of the slab, accurately reproduces the observed strong asymmetry of the line shape. Using this model, we extract from our data a shift of the bulk atomic medium resonance, which oscillates with the thickness of the medium. The origin of this oscillation is not understood, and we have formulated a few directions that should be explored theoretically.

The data presented in this Letter can be found in Ref. [43].

We acknowledge fruitful discussions with J. Ruostekoski. T.P. is supported by the Délégation Générale de l'Armement-Defence Science and Technology Laboratory fellowship 2015600028. We also acknowledge financial support from Engineering and Physical Sciences Research Council (Grant No. EP/ L023024/1) and Durham University.

[1] M. Gross and S. Haroche, Superradiance: An essay on the theory of collective spontaneous emission, Phys. Rep. 93, 301 (1982).

[2] W. Guérin, M. T. Rouabah, and R. Kaiser, Light interacting with atomic ensembles: Collective, cooperative and mesoscopic effects, J. Mod. Opt. 64, 895 (2017).

[3] D. E. Chang, J. Ye, and M. D. Lukin, Controlling dipoledipole frequency shifts in a lattice-based optical atomic clock, Phys. Rev. A 69, 023810 (2004).

[4] S. L. Bromley et al., Collective atomic scattering and motional effects in a dense coherent medium, Nat. Commun. 7, 11039 (2016).
[5] S. L. Campbell et al., A Fermi-degenerate threedimensional optical lattice clock, Science 358, 90 (2017).

[6] R. J. Bettles, S. A. Gardiner, and C. S. Adams, Cooperative ordering in lattices of interacting two-level dipoles, Phys. Rev. A 92, 063822 (2015).

[7] R. J. Bettles, S. A. Gardiner, and C. S. Adams, Enhanced Optical Cross Section via Collective Coupling of Atomic Dipoles in a 2D Array, Phys. Rev. Lett. 116, 103602 (2016).

[8] E. Shahmoon, D. S. Wild, M. D. Lukin, and S. F. Yelin, Cooperative Resonances in Light Scattering from TwoDimensional Atomic Arrays, Phys. Rev. Lett. 118, 113601 (2017).

[9] J. Perczel, J. Borregaard, D. E. Chang, H. Pichler, S. F. Yelin, P. Zoller, and M. D. Lukin, Topological Quantum Optics in Two-Dimensional Atomic Arrays, Phys. Rev. Lett. 119, 023603 (2017).

[10] R. Friedberg, S. R. Hartmann, and J. T. Manassah, Frequency shift and absorption by resonant systems of two-level atoms, Phys. Rep. 7, 101 (1973).

[11] R. Röhlsberger, K. Schlage, B. Sahoo, S. Couet, and R. Roeffer, Collective Lamb shift in single-photon superradiance, Science 328, 1248 (2010).

[12] J. Keaveney, A. Sargsyan, U. Krohn, I. G. Hughes, D. Sarkisyan, and C. S. Adams, Cooperative Lamb Shift in an Atomic Vapor Layer of Nanometer Thickness, Phys. Rev. Lett. 108, 173601 (2012).

[13] J. Javanainen and J. Ruostekoski, Light propagation beyond the mean-field theory of standard optics, Opt. Express 24, 993 (2016).

[14] J. Javanainen, J. Ruostekoski, Y. Li, and S.-M. Yoo, Exact electrodynamics versus standard optics for a slab of cold dense gas, Phys. Rev. A 96, 033835 (2017).

[15] J. Javanainen, J. Ruostekoski, Y. Li, and S.-M. Yoo, Shifts of a Resonance Line in a Dense Atomic Sample, Phys. Rev. Lett. 112, 113603 (2014).

[16] S. J. Roof, K. J. Kemp, M. D. Havey, and I. M. Sokolov, Observation of Single-Photon Superradiance and the Cooperative Lamb Shift in an Extended Sample of Cold Atoms, Phys. Rev. Lett. 117, 073003 (2016).

[17] L. Corman, J.-L. Ville, R. Saint-Jalm, M. Aidelsburger, T. Bienaimé, S. Nascimbéne, J. Dalibard, and J. Beugnon, Transmission of near-resonant light through a dense slab of cold atoms, Phys. Rev. A 96, 053629 (2017).

[18] H. Fearn, D. F. V. James, and P. Milonni, Microscopic approach to reflection, transmission and the Ewald-Osen extinction theorem, Am. J. Phys. 64, 986 (1996).

[19] R. P. Feynman, R. B. Leighton, and M. Sands, Lectures on Physics (Addison-Wesley, Reading, MA, 2006), Vol. 1, Chap. 30.

[20] J. D. Jackson, Classical Electrodynamics (Wiley, New York, 1998).

[21] See Supplemental Material at http://link.aps.org/ supplemental/10.1103/PhysRevLett.120.243401 for more details about the cavity model used in Ref. [12] and in this work, and about the way error bars are calculated in Figs. 3 (a) and 3(b), which includes Refs. [22,23].

[22] G Brooker, Modern Classical Optics (Oxford University, New York, 2002). 
[23] J. Keaveney, I. G. Hughes, A. Sargsyan, D. Sarkisyan, and C. S. Adams, Maximal Refraction and Superluminal Propagation in a Gaseous Nanolayer, Phys. Rev. Lett. 109, 233001 (2012).

[24] D. Sarkisyan, T. Varzhapetyan, A. Sarkisyan, Y. Malakyan, A. Papoyan, A. Lezama, D. Bloch, and M. Ducloy, Spectroscopy in an extremely thin vapor cell: Comparing the cell-length dependence in fluorescence and in absorption techniques, Phys. Rev. A 69, 065802 (2004).

[25] To avoid a cavity effect from the sapphire plates, they form a wedge with an angle of $10 \mathrm{mrad}$, much larger than the $0.1 \mathrm{mrad}$ angle of the atomic slab.

[26] A. Sargsyan, A. Tonoyan, G. Hakhumyan, C. Leroy, Y. Pashayan-Leroy, and D. Sarkisyan, Complete hyperfine Paschen-Back regime at relatively small magnetic fields realized in potassium nano-cell, Europhys. Lett. 110, 23001 (2015).

[27] E. Jahier, J. Guéna, P. Jacquier, M. Lintz, A. V. Papoyan, and M. A. Bouchiat, Temperature-tunable sapphire windows for reflection loss-free operation of vapor cells, Appl. Phys. B 71, 561 (2000).

[28] G. Truong, J. D. Anstie, E. F. May, T. M. Stace, and A. N. Luiten, Absolute absorption line-shape measurements at the shot-noise limit, Phys. Rev. A 86, 030501 (2012).

[29] I. Maurin, P. Todorov, I. Hamdi, A. Yarovitski, G. Dutier, D. Sarkisyan, S. Saltiel, M.-P. Gorza, M. Fichet, D. Bloch, and M. Ducloy, Probing an atomic gas confined in a nanocell, J. Phys. Conf. Ser. 19, 20 (2005).

[30] M. F. H. Schuurmans, Spectral narrowing of selective reflection, J. Phys. (Paris) 37, 469 (1976).

[31] T. A. Vartanyan and D. L. Lin, Enhanced selective reflection from a thin layer of a dilute gaseous medium, Phys. Rev. A 51, 1959 (1995).

[32] B. Zambon and G. Nienhuis, Reflection and transmission of light by thin vapor layers, Opt. Commun. 143, 308 (1997).
[33] G. Dutier, S. Saltiel, D. Bloch, and M. Ducloy, Revisiting optical spectroscopy in a thin vapor cell: Mixing of reflection and transmission as a Fabry-Perot microcavity effect, J. Opt. Soc. Am. B 20, 793 (2003).

[34] M. A. Zentile, J. Keaveney, L. Weller, D. J. Whiting, C. S. Adams, and I. G. Hughes, ElecSus: A program to calculate the electric susceptibility of an atomic ensemble, Comput. Phys. Commun. 189, 162 (2015).

[35] By performing the integration over the velocity distribution, we have checked that it indeed has no effect.

[36] Although the temperature is left as a free parameter here, the fitted values are very close to the values measured on the experiment by the thermocouple in contact with the reservoir.

[37] K. A. Whittaker, J. Keaveney, I. G. Hughes, A. Sargsyan, D. Sarkisyan, and C.S. Adams, Optical Response of GasPhase Atoms at Less than $\lambda / 80$ from a Dielectric Surface, Phys. Rev. Lett. 112, 253201 (2014).

[38] L. Weller, R. J. Bettles, P. Siddons, C. S. Adams, and I. G. Hughes, Absolute absorption on the rubidium D1 line including resonant dipole-dipole interactions, J. Phys. B 44, 195006 (2011).

[39] P. Todorov and D. Bloch, Testing the limits of the Maxwell distribution of velocities for atoms flying nearly parallel to the walls of a thin cell, J. Chem. Phys. 147, 194202 (2017).

[40] G. Dutier, A. Yarovitski, S. Saltiel, A. Papoyan, D. Sarkisyan, D. Bloch, and M. Ducloy, Collapse and revival of a Dicke-type coherent narrowing in a sub-micron thick vapor cell transmission spectroscopy, Europhys. Lett. 63, 35 (2003).

[41] O. Morice, Y. Castin, and J. Dalibard, Refractive index of a dilute Bose gas, Phys. Rev. A 51, 3896 (1995).

[42] J. Ruostekoski and J. Javanainen, Quantum field theory of cooperative atom response: Low light intensity, Phys. Rev. A 55, 513 (1997).

[43] See DOI: 10.15128/r11z40ks81r. 